Eurasian Journal of
Biological and Chemical Sciences
EJBCS
Journal homepage: www.dergipark.org.tr/ejbcs

\title{
Synthesis of Biologically Active 4-Arylideneamino-2,4-dihydro-3H-1,2,4-triazol-3- one Compound by Ultrasonic Radiation and Conventional Method, Comparison of Methods and Elucidation of Structure
}

\author{
İnci Selin DOĞAN ${ }^{1 * \mathbb{D}}$, Bahittin KAHVECí ${ }^{2}$ (D) \\ ${ }^{{ }^{*} 1}$ Karadeniz Technical Uni., Faculty of Pharmacy, Department of Pharmaceutical Chemistry, Trabzon, Turkey. \\ ${ }^{2}$ Karadeniz Technical Uni., Faculty of Health Sciences, Department of Nutrition and Dietetics, Trabzon, Turkey.
}

\begin{abstract}
Today, the majority of the drugs used in the treatment of diseases are obtained by synthesis. A large proportion of the compounds have obtained heterocyclic structure. Therefore, synthesis of new heterocyclic compounds has always attracted and continues to attract scientist. It is known that compounds bearing triazole skeleton in the heterocyclic have an important place.

The reactions of 4-amino-triazole compounds, especially with aromatic aldehydes, have been known for a long time and this reaction is carried out by conventional heating methods. However, the development of alternative heating methods to these conventional methods has also recently been studied. One of the most important of these is organic synthesis by microwave method and it is widely used. Another alternative method that has recently been used in organic synthesis is the realization of organic synthesis reactions with ultrasonic radiation. Ultrasound-assisted organic synthesis is an environmentally friendly, modern and economical method used to accelerate reactions.

In this study, optimum conditions were determined for this reaction by using ultrasonic radiation method and the results were compared with the classical method. For this purpose, in the first step iminoester was synthesized by using Pinner method. The reaction of this iminoester with ethylcarbazate gave the corresponding hydrazone. Then the reaction of this obtained hydrazone derivative with hydrazine hydrate, the 4-amino-1,2,4-triazole derivative compound was synthesized. In the final reaction step, the interaction of this amino compound with 3-bromo-4-fluorobenzaldehyde was carried out by conventional and novel method (ultrasonic radiation) to obtain the targeted imine compound. The chemical structure of the new compound will be determined by spectroscopic methods such as ${ }^{1} \mathrm{H}$ - and ${ }^{13} \mathrm{C}-\mathrm{NMR}$ and IR.
\end{abstract}

The results compared in terms of reaction time, yield and purity between conventional and ultrasonic method. And we found that the ultrasonic radiation method is much more advantageous.

Keywords: 1,2,4-triazole, Ultrasonic Radiation Method, Iminoester, Schiff base, Benzaldehyde, Conventional Method.

(c) EJBCS. All rights reserved.

\section{Introduction}

It is known that compounds bearing triazole skeleton in the heterocyclic chemical have an important place. Therefore, studies on the synthesis of these systems have become particularly popular recently. The reactions of 4-aminotriazole compounds, especially with aromatic aldehydes, have been known for a long time and this reaction is carried out by conventional heating methods (Kahveci et al., 2000).

However, an experimental study on the realization of this reaction using ultrasonic radiation in these systems carried out with this study and the results compared with the classical method.
For this purpose, 4-amino-1,2,4-triazole compound registered in the literature (Kahveci et al., 2000) have been synthesized and the reaction of this compound with 3 bromo-4-fluorobenzaldehyde have investigated by both classical and ultrasonographic methods and a novel Schiff base derivative compound was obtained.

Ultrasound-assisted organic synthesis (Sonochemistry) is an environmentally friendly, modern and economical method used to accelerate reactions (Alissa, 2014). Ultrasound can increase chemical reactivity besides heat, pressure, light and catalyst. Application of power of sound to chemical processes: 1 -Ultrasound can increase chemical 
reactivity besides heat, pressure, light and catalyst; 2Chemical changes by power of ultrasound lower frequency as result of generated cavitation (Sarpong, 2003; Mason et al.,2002).

When we look at the benefits of using ultrasonic radiation in chemical synthesis (Sonochemistry); the reaction rate increases and allows the reaction to proceed in mild conditions, it shortens the onset time for exothermic reactions, reactions can usually walk without need for additives as a catalyst, the number of reaction steps and duration decreases, reaction can be directed to alternative pathway (Thompson et al., 1999; Gogate et al., 2006; Url1, 2020).

Sonochemistry can use for; synthesis processing in nanomaterials; cancer treatment, drug delivery in therapeutic ultrasound; mizing, preservation, extraction in food technology; electroplating, electrosynthesis, electroanalysis in electrochemistry; chemical synthesis, catalysis in Green Chemistry (Thompson et al., 1999; Gogate et al., 2006; url-1, 2020).

Therefore, here we aimed to analyze the utility of Sonochemistry method for the synthesis procedure.

In this study, we aimed to synthesize a novel Schiff base derivative "4-(3-bromo-4-fluorobenzylidenamino)-5-(3bromobenzyl)-2H-1,2,4-triazol-3(4H)-on" compound by both Conventional and Ultrasonic radiation method (Sonochemistry), analyze the structure by spectral methods ( ${ }^{1} \mathrm{H}$ - and ${ }^{13} \mathrm{C}-\mathrm{NMR}$ and IR) and compare the methods by providing optimum conditions.

\section{Materials and Method}

All the chemicals purchased from Merck, Fluka AG, and Sigma-Aldrich. Merck Kieselgel60 F254 plates were used for thin layer chromatography (TLC) to monitor reactions. Melting points (m.p.) were determined using an Electrothermal 9200 capillary melting point apparatus and are uncorrected. ${ }^{1} \mathrm{H}-\mathrm{NMR} \quad(400 \mathrm{MHz})$ and ${ }^{13} \mathrm{C}-\mathrm{NMR}$ $(100 \mathrm{MHz})$ spectra were recorded with Brucker Avonce Ultrashield NMR spectrometers, and Elemental analyses were performed by Leco CHNS-932 LECO932CHNS elemental analysis apparatus.

General experimental procedure for synthesis of the compounds

\section{1) Synthesis of Iminoester Hydrochloride (Compound 1a)}

The iminoester hydrochlorides used as starting compounds of our study were synthesized according to the Pinner Method recorded in the literature (Figure 1) (Pinner, 1892). In this method, suitable nitrile derivatives and ethanol were reacted with $\mathrm{HCl}(\mathrm{g})$ at anhydrous ether at $0-5^{\circ} \mathrm{C}$ to obtain the corresponding iminoester hydrochloride.

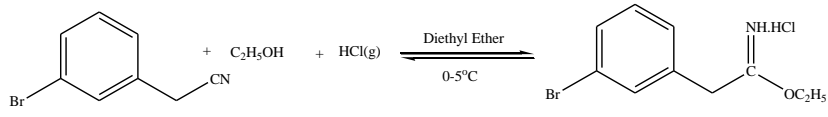

Compound 1a

Figure 1. Synthesis of Iminoester Hydrochloride Derivative Compound (1a) by Pinner Method

For the production of $\mathrm{HCl}$ gas, $\mathrm{H}_{2} \mathrm{SO}_{4}$ and $\mathrm{NH}_{4} \mathrm{Cl}$ were reacted and the $\mathrm{HCl}$ gas formed was passed to the reaction medium with the Kip device. The reaction setup is given in figure 2 .

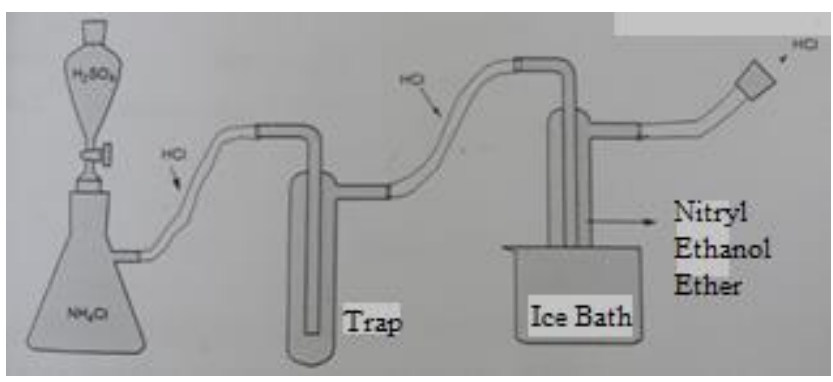

Figure 2. Kip setup for obtaining $\mathrm{HCl}(\mathrm{g})$

2) Synthesis of Esther ethoxycarbonyl hydrazone derivative (Compound 2a)

The ester ethoxycarbonyl hydrazone derivatives to be used in the study for the production of triazole derivatives were synthesized according to the method in the literature (Figure 3) (İkizler et al., 1996). In this method; the iminoester hydrochlorides and ethyl carbazate were dissolved in ethanol and reacted in a sealed flask. The flask was placed in the ice bath and mixing was done with the help of a magnetic stirrer.

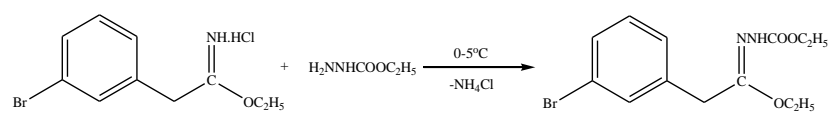

Compund 2a

Figure 3. Synthesis of Ester ethoxycarbonyl hydrazone derivative compound (2a)

\section{3) Synthesis of 4-Amino-1,2,4-triazole derivative (Compound 3a)}

The corresponding 4-Amino-1,2,4-triazole compound was obtained from the reaction of esther ethoxycarbonyl hydrazone with hydrazine hydrate (Figure 4) (Kahveci, 2005).

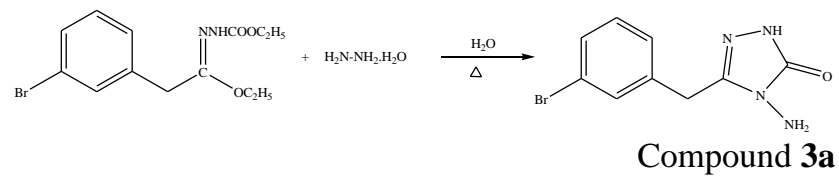

Figure 4. Synthesis of 4-Amino-1,2,4-triazole derivative compound (3a) 


\section{3) Synthesis of Schiff base derivative final compound (Compound I)}

The reaction of amino-triazole compound with 3-Bromo-4fluorobenzaldehyde, corresponding Schiff base derivative was synthesized by two different methods (Figure 5).

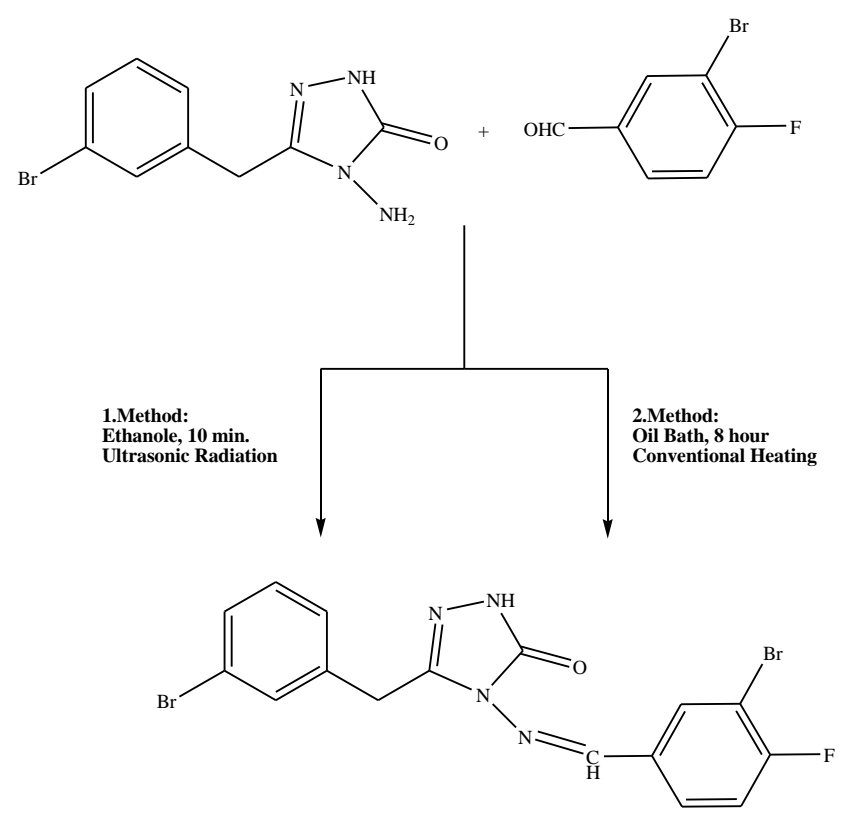

Figure 5. Synthesis of Schiff base derivative compound I by two different methods

\section{Results}<smiles>CCOC(=N)Cc1cccc(Br)c1</smiles>

Absolute ethanol $(5.9 \mathrm{ml}, 0.1 \mathrm{~mol})$ was added to the solution of 4-bromobenzylonitrile (10.3 ml, $0.1 \mathrm{~mol})$ with anhydrous ether $(10 \mathrm{ml})$. Dry $\mathrm{HCl}$ gas was passed through the mixture until saturation. The mixture was left in the deep cooler for 12 hours and precipitated by the addition of anhydrous ether. The crystals formed were filtered, washed with anhydrous ether and dried over $\mathrm{CaCl}_{2}$. The product obtained was identified as Compound 1a (Mp: $\left.85^{\circ} \mathrm{C}\right)$. Yield: $9.5 \mathrm{~g}$ $(51.21 \%)$<smiles>CCOC(=O)N=C(Cc1cccc(Br)c1)OCC</smiles>

The absolute ethanol solution of imino 4-bromobenzyl ethyl ester hydrochloride (Compound 1a, $6 \mathrm{~g}, 0.03 \mathrm{~mol}$ ) was added to the solution of ethyl carbazate $(3.15 \mathrm{~g}, 0.03 \mathrm{~mol})$ in absolute ethanol. The mixture was reacted in a sealed flask. The flask placed in the ice bath was mixed with the help of a magnetic stirrer. A white turbidity occurred in the reaction medium during the reaction, because of the formation of $\mathrm{NH}_{4} \mathrm{Cl}$ salt in the reaction medium precipitates. After the reaction was over, $\mathrm{NH}_{4} \mathrm{Cl}$ in the medium was filtered. The mixture was kept in the freezer for 12 hours.
Then the solvent was blown off in the evaporator. After extraction with petroleum ether, the crystals formed were filtered, washed with anhydrous ether and dried over $\mathrm{CaCl}_{2}$. The product obtained was identified as Compound 2a (Mp. 85-86 $\left.{ }^{\circ} \mathrm{C}\right)$. Yield: $1.5 \mathrm{~g}(20 \%)$.<smiles>Nn1c(Cc2cccc(Br)c2)n[nH]c1=O</smiles>

The corresponding 4-Amino-1,2,4-triazole compound was obtained from the reaction of the esther ethoxycarbonyl hydrazone with hydrazine hydrate and Compound 3a obtained (Mp. $\left.85-86^{\circ} \mathrm{C}\right)$. Yield: $1.5 \mathrm{~g}(20 \%)$.

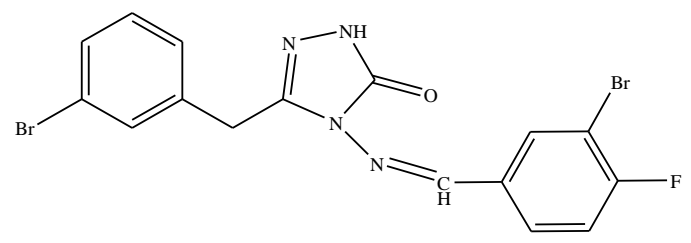

4-(3-bromo-4-flurobenzylidenamino)-5-(3-bromobenzyl)2H-1,2,4-triazol-3(4H)-on

Method 1: 0.01 mol 5-(3-bromobenzyl)-4-amino-2H-1,2,4triazole-3(4H)-on (Compound 3a) was added to $0.01 \mathrm{~mol}$ of 3-bromo-4-flurobenzaldehyde derivative, the mixture was heated dry to dry in an oil bath for 1.5 hours. The reaction process is controlled by TLC. Water is added to the final mixture. The precipitate is filtered and purified by crystallization from the ethanol-water mixture (conventional method, İkizler et al, 1991).

Method 2: 0.01 mol 5-(3-bromobenzyl)-4-amino-2H-1,2,4triazole-3(4H)-one (Compound 3a) was added to $0.01 \mathrm{~mol}$ of 3-bromo-4-flurobenzaldehyde dissolved in ethanol (1-2 drops of acetic acid is added) and mixture was heated in the ultrasonic bath for 10 minutes using the ultrasonic radiation $\left(\right.$ at $50^{\circ} \mathrm{C}$ ). The reaction process is controlled by TLC. Water is added to the final mixture. The precipitate is filtered and purified by crystallization from the appropriate ethanolwater mixture (ultrasonic radiation method).

4-(3-bromo-4-flurobenzylideneamino)-5-(3-bromobenzyl)2H-1,2,4-triazole-3(4H)-one (Compound I);

Yield \% 90/96 (Method I/II); Mp.: 213-214 ${ }^{\circ} \mathrm{C}$. Analysis (\% Calculated/found) for $\mathrm{C}_{16} \mathrm{H}_{11} \mathrm{Br}_{2} \mathrm{FN}_{4} \mathrm{O}$ (Mw 426.09) $\mathrm{C}$ : 45.10/45.01, H: 2.60/2.53, N: 13.15/13.09

IR spectrum (v): 3173 (N-H stretching), 3055 (aromatic C$\mathrm{H}$ stretching), $1700(\mathrm{C}=\mathrm{O}$ stretching, ketone), $1587(\mathrm{C}=\mathrm{N}$ stretching), 1053 (C-F), 815 (C-Br), 743 and $714 \mathrm{~cm}-1$ (monosubstitue benzene deformation) bands.

${ }^{1} \mathrm{H}-\mathrm{NMR}$ spectrum (400 MHz, DMSO-d 6 ) ( $\delta$ ): 4.068 (s, 2H, $\left.-\mathrm{CH}_{2-}\right), 7.229-8.085$ ( $\mathrm{m}, 7 \mathrm{H}$, phenyl ring protons), 9.638 (s, $1 \mathrm{H},-\mathrm{N}=\mathrm{CH}-), 12.015 \mathrm{ppm}\left(\mathrm{s}, 1 \mathrm{H}, \mathrm{N}_{1}-\mathrm{H}\right)$ peaks.

${ }^{13} \mathrm{C}-\mathrm{NMR}$ spectrum $\left(100 \mathrm{MHz}, \mathrm{DMSO}-\mathrm{d}_{6}\right)(\delta): 31.03\left(\mathrm{CH}_{2}\right)$, 109.37-146.142 (Ar C-H), $151.142(\mathrm{C}=\mathrm{N}), 151.198$ (C5triazole), $159.062(\mathrm{C}=\mathrm{O}), 161.55 \mathrm{ppm}(\mathrm{C}-\mathrm{F})$ peaks. 


\section{Discussion}

In this study, the synthesis of triazole-imine derivative compounds with conventional and ultrasonic radiation was aimed and a compound (Compound I) was synthesized in this study.

For this, firstly, starting materials, iminoester hydrochlorides (Compound 1a) were synthesized according to the Pinner method (Figure 1) (Pinner, 1892). In the second stage, the ethyl carbazate compound and iminoester hydrochlorides were reacted under suitable conditions in ethanol to obtain ester ethoxycarbonyl hydrazones (Compound 2a) according to the literature (Figure 3) (İkizler et al., 1996). In the third step, the corresponding 4amino-1,2,4-triazole-5-one derivative compound (Compound 3a) was obtained from the reaction of the ester ethoxycarbonyl hydrazones with hydrazine hydrate (Figure 4) (Kahveci, 2005).

In the last step, comparisons were made using 2 different methods. While conventional heating method is used in method 1 (Íkizler et al., 1991), ultrasonic radiation method is used in method 2. Synthesis of the resulting product triazole imine derivative (Compound I) was carried out by both methods.

The physical properties of the compounds and Rf values in the solvent system were determined in thin layer chromatography. The structures of the synthesized compounds were analyzed by spectroscopic methods such as IR, ${ }^{1} \mathrm{H}-\mathrm{NMR},{ }^{13} \mathrm{C}-\mathrm{NMR}$ and elemental analysis.

The spectral data (IR, ${ }^{1} \mathrm{H}-\mathrm{NMR},{ }^{13} \mathrm{C}-\mathrm{NMR}$ ) of the compounds are in accordance with the literature (Layer, 1963; Silverstein et al., 1998; Pretsch et al., 1983).

In the reaction by the conventional method (method 1), the compounds were mixed for 1,5 hours in the oil bath at the boiling temperature. In method 2 , in the reaction, the compounds were mixed for 10 minutes in the ultrasonic bath using the ultrasonic radiation heating. The reaction time was reduced from 1,5 hours to 10 minutes in method 2 .

Looking at the reaction efficiencies, the resulting imine compound was obtained with a yield of $90 \%$ with method 1, while with a yield of $96 \%$ in method 2 .

When we compare the methods in terms of reaction time, efficiency and energy efficiency, we found that the ultrasonic radiation method is much more advantageous.

\section{Conclusion}

As a Conclusion, this is the first attempt to synthesize Schiff bases of 4-Amino-triazoles by Ultrasonic radiation method. The most important advantage of this experiment over other methods is short reaction time and economical in terms of energy spent. In terms of efficiency, it shows a competitive feature to other methods. The study promotes later ultrasonic assisted synthesis studies on these heterocyclic aromatic systems.

\section{Acknowledgements}

Names of the funding organisation(s) should be given. If no The synthesized work was supported by Karadeniz Technical University Research Project (BAP-02-6035).

\section{Authors' contributions:}

İ.S.D. and B.K. conceived, designed and performed the experiment; İ.S.D. and B.K. wrote the manuscript.

\section{Conflict of interest disclosure:}

There was no conflict of interest.

\section{References}

Alissa SA. 2014. Ultrasound synthesis of five membered heterocycles. Chem Sci Rev Let. 3(12):1219-1236.

Gogate P, Pandit, AB, Tayal RK. 2006. Cavitation: A technology on the horizon. Current Science. 91(1):35-46.

https://www.slideshare.net/wimbaji/ultrasonicapplication-in-chemical-engineering-presentation [20.02.2020].

İkizler A, Demirbaş N, Demirbaş A, İkizler AA. 1996. The reaction of Ester Etohoxycarbonyl hydrazones with carboxylic acid hydrazides. Polish J Chem. 70:1114-1120.

İkizler AA, Yildırım N. 1991. Synthesis of some benzylidenamino compounds. Monatsch Chem. 122:557563.

Kahveci B, İkizler A. 2000. Convenient synthesis of some 4-(Alkylamino)-4,5-dihydro-1 $\mathrm{H}$-1,2,4-triazol-5-ones. Turk J Chem. 24:343- 351.

Kahveci B. 2005. Synthesis of 4-amino-4,5-dihydro$1 \mathrm{H}$-1,2,4-triazole-5-ones and their isatin-3-imine derivatives. Molecules. 10:376-382.

Layer RW. 1963. The chemistry of imines. Chemical Reviews. 63:489-510.

Mason TJ, Lorimer JP. 2002. Applied Sonochemistry: Uses of Power Ultrasound in Chemistry and Processing. By Timothy J Mason and John P Lorimer, Wiley-VCH Verlag, Weinheim, 2002, 303 pp, ISBN 3-527-30205-0

Pinner A. 1892. Die imidoether und ihne Derivate, 1.Auflaze, Oppenheim, Berlin.

Pretsch E, Clerk T, Seibl J, Simon W. 1983. Tables of spectral data for structure determination of organic compounds. Berlin: Springer-Verlag.

Sarpong R. 2003. Ultrasound Applications in Organic synthesis, Stoltz Literature Meeting.

Silverstein RM, Webster FX. 1998. Spectrometric identification of organic compounds. New York: John Wiley \& Sons Inc.

Thompson LH, Doraiswamy LK. 1999. Sonochemistry: Science and Engineering. Ind Eng Chem Res. 38(4):12151249. 